\title{
An Energy-Saving Routing Algorithm for Wireless Body Sensor Network using Data Compression Technique
}

\author{
Karthick .K \\ Department of CSE \\ PG Scholar, \\ Sri Ramakrishna Engineering College, \\ Coimbatore-641 022.
}

\author{
R. Vijaya Kumar \\ (Assistant Professor), \\ Department of CSE (UG), \\ Sri Ramakrishna Engineering College, \\ Coimbatore-641 022.
}

\begin{abstract}
A wireless sensor network (WSN) is used to monitor the environmental condition. There consist of $\mathrm{N}$ number of sensor nodes which are connected to each other and they are clustered in to different groups. There have a cluster head $(\mathrm{CH})$ which is used to manage the cluster members $(\mathrm{CM})$. when the sensor node is active, energy will be reduced vitally in order to increase the life time of the sensor node different application has be implemented .In this method Energy consumption will been reduced by implementing leach-c protocol and LZW compression technique so the lifetime of the sensor node is increased.
\end{abstract}

\section{INTRODUCTION}

\subsection{Wireless networks}

A wireless network is used to connect two or more nodes in a typical manner which is used to reduce the installation charge when compared with the wired network. Wireless telecommunications networks are implemented using radio communication. This implementation takes place at the physical level (layer) of the OSI model network structure.

\subsection{Wireless Sensor Network}

A wireless sensor network consist of sensor nodes which is used to monitor physical and environment condition simultaneously, such as temperature, pressure, sound and etc. Firstly it has been developed for the military application then it has been used in healthcare applications, traffic control and etc.

These sensor node consist of radio transceiver, microcontroller and a battery. The size of the sensor node is tiny and its cost depend on the size.

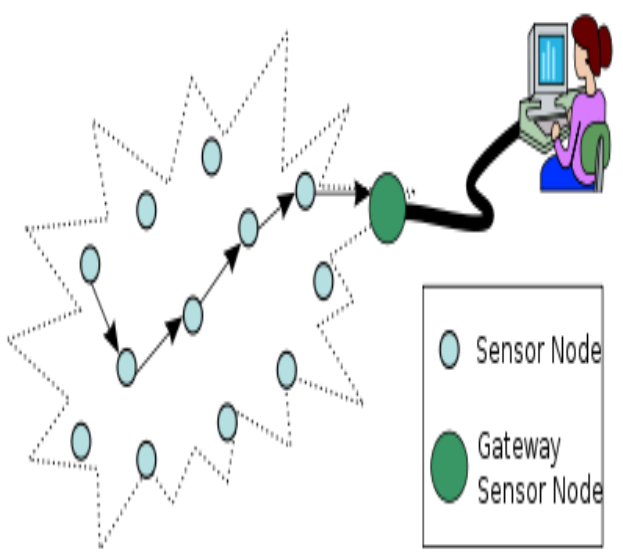

Fig 1: Wireless sensor network

\section{SYSTEM MODULE}

\subsection{Wireless Sensor Network Node} Creation.

Wireless sensor network consists of $\mathrm{N}$ number of the sensor node these sensor node has been placed in the different geographical location the energy resource they have been used is the battery. With the help of GPS the location of sensor node will be identified by the base station. The battery cannot be replaced frequently because they have been installed in dense forest or some reserved area. Energy plays a vital role in the sensor node so different protocols have been implemented. These protocols helps to increase and prolong the life time of the sensor node.

In LEACH's distributed cluster formation algorithm, each and every node takes their own decisions because of this there obtain a poor cluster setup which will not help is energy saving. In order to overcome this a new protocol called LEACH-C(LEACH-Centralized) has been introduced.

In LEACH-C protocol the cluster head $(\mathrm{CH})$ has been selected based on energy level of sensor node. The sensor node with higher energy will be represented as the cluster head others are considered as the cluster member. Here they perform a randomized rotation of cluster head based on the energy level. If the energy level of the $\mathrm{CH}$ is reduced in the cluster group then it will search for the other sensor node with higher energy in the given respective group. If it finds any of the cluster member with higher energy it will be converted as the cluster head $(\mathrm{CH})$. Thus the life time of the cluster head will always prolong.

There consist of two phase called as the setup phase and steady-state phase, these phase helps in initial setup of the sensor node.

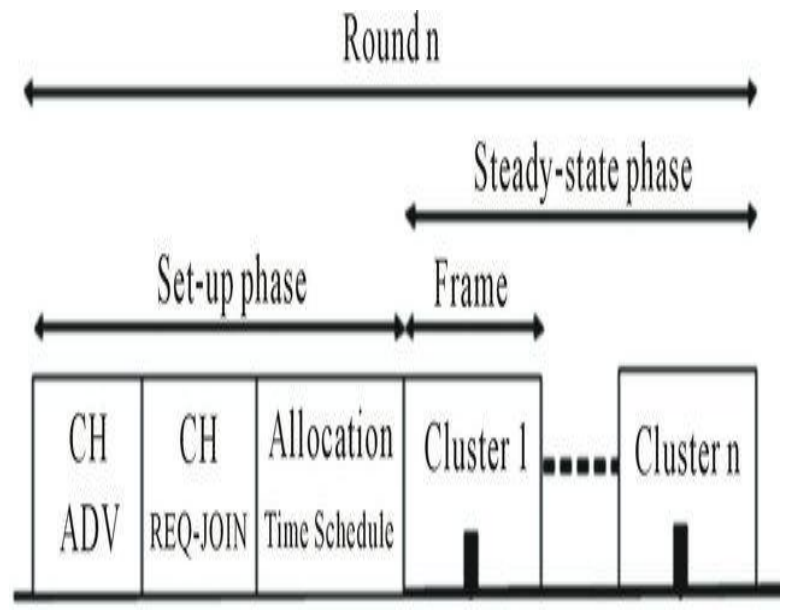

Fig 2: Schedule of set-up and steady-state phase in a round 


\section{Setup Phase}

When the node has been selected as the cluster head, it will send an advertisement message to all other cluster members stating that it is a $\mathrm{CH}$. The non-cluster head or cluster member (CM) receives the advertisement message .Then $\mathrm{CM}$ will be send the join request to the cluster head $(\mathrm{CH})$

The cluster member with join packet contains the ID, this ID will be sent to the cluster head .thus the cluster head creates the TDMA schedule then it will broadcast the TDMA table to the cluster members.

\section{Steady-state phase}

In fig 2 the data transmission happens and data's have been sent to the cluster head based on the time slot TDMA one of the major advantage in this concept is that the data collision will not occur. The energy loss will occur only if data collision happen. Due to this TDMA sends the data in a proper scheduling

After implementing leach-c protocol with TDMA large amount of energy have been saved and thus the life time of the sensor node has been prolonged

\subsection{LEACH-C : Base Station Cluster \\ Formation}

In LEACH's distributed cluster formation algorithm, each and every node takes their own decisions because of this there obtain a poor cluster setup which will not help is energy saving. In order to overcome this a new protocol called LEACH-C (LEACH-Centralized) has been introduced.

In LEACH-C protocol the cluster head $(\mathrm{CH})$ has been selected based on energy level of sensor node. The sensor node with higher energy will be represented as the cluster head others are considered as the cluster member. Here they perform a randomized rotation of cluster head based on the energy level. If the energy level of the $\mathrm{CH}$ is reduced in the cluster group then it will search for the other sensor node with higher energy in the given respective group. If it finds any of the cluster member with higher energy it will be converted as the cluster head $(\mathrm{CH})$. Thus the life time of the cluster head will always prolong.

The life time of the cluster head is increased due to the randomized rotation of the cluster. Based on the energy level the cluster head is selected. The sensor node with higher energy is converted as the cluster head.

\subsection{LEMPEL-ZIV- \\ ELCH (LZW) Algorithm}

\subsubsection{Compression Algorithm}

Lempel-Ziv-Welch (LZW) is a lossless data compression algorithm. This algorithm can be implemented in a very simple format, they play a vital role in data compression technique.

\subsubsection{Encoding}

This is a lossless data compression technique in which its very simple to implement firstly the given data can be in different format, consider it is in a character format. Initially these characters will be grouped then each and every character will be compressed. In case of compression they will be converting character string in to the binary value (e.g. 10000100011000100) in such a way the data's will be compressed .These compression will be in form of more secured way. All data's transferred from cluster member to the cluster head have been compressed and sent to the cluster head $(\mathrm{CH})$. In cluster head uses only less amount of energy to transfer the given data.

\subsubsection{Decoding}

In case of decoding the compressed data will be transferred from the cluster head to the base station. The base station will receive all the compressed data and perform the decoding process. Here the received binary data will be converted in to the character or string.

After undergoing all these process we will get the actual data which is sent from the cluster member. The main aim of this compression is to prolong the lifetime of the cluster head $(\mathrm{CH})$.

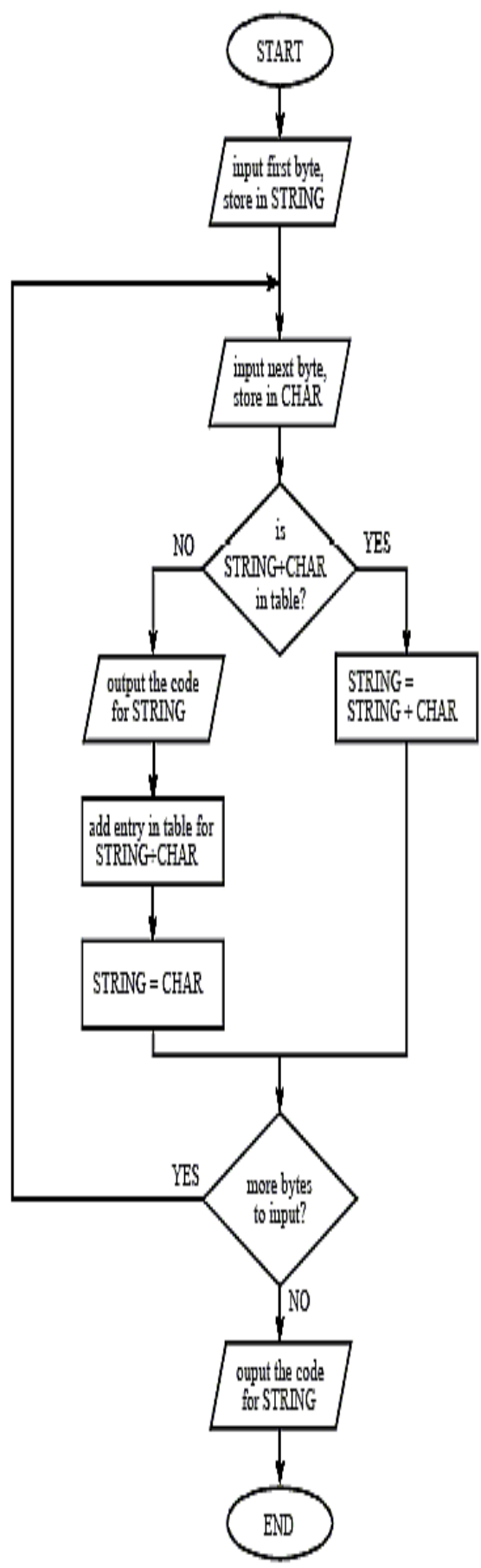

Fig 3: LZW compression flowchart 
This compression is a lossless compression so that it is very simple to implement using Lempel-Ziv-Welch (LZW) algorithm they have been widely used in different area.

Here's what it might look like in pseudo code:

string s;

char ch;

$\mathrm{s}=$ empty string;

while (there is still data to be read)

$\mathrm{ch}=$ read a character;

if (dictionary contains $\mathrm{s}+\mathrm{ch}$ )

else

$$
\mathrm{s}=\mathrm{s}+\mathrm{ch}
$$$$
\{
$$

encode $s$ to output file; add s+ch to dictionary; $\mathrm{s}=\mathrm{ch}$;

\}

encode s to output file;

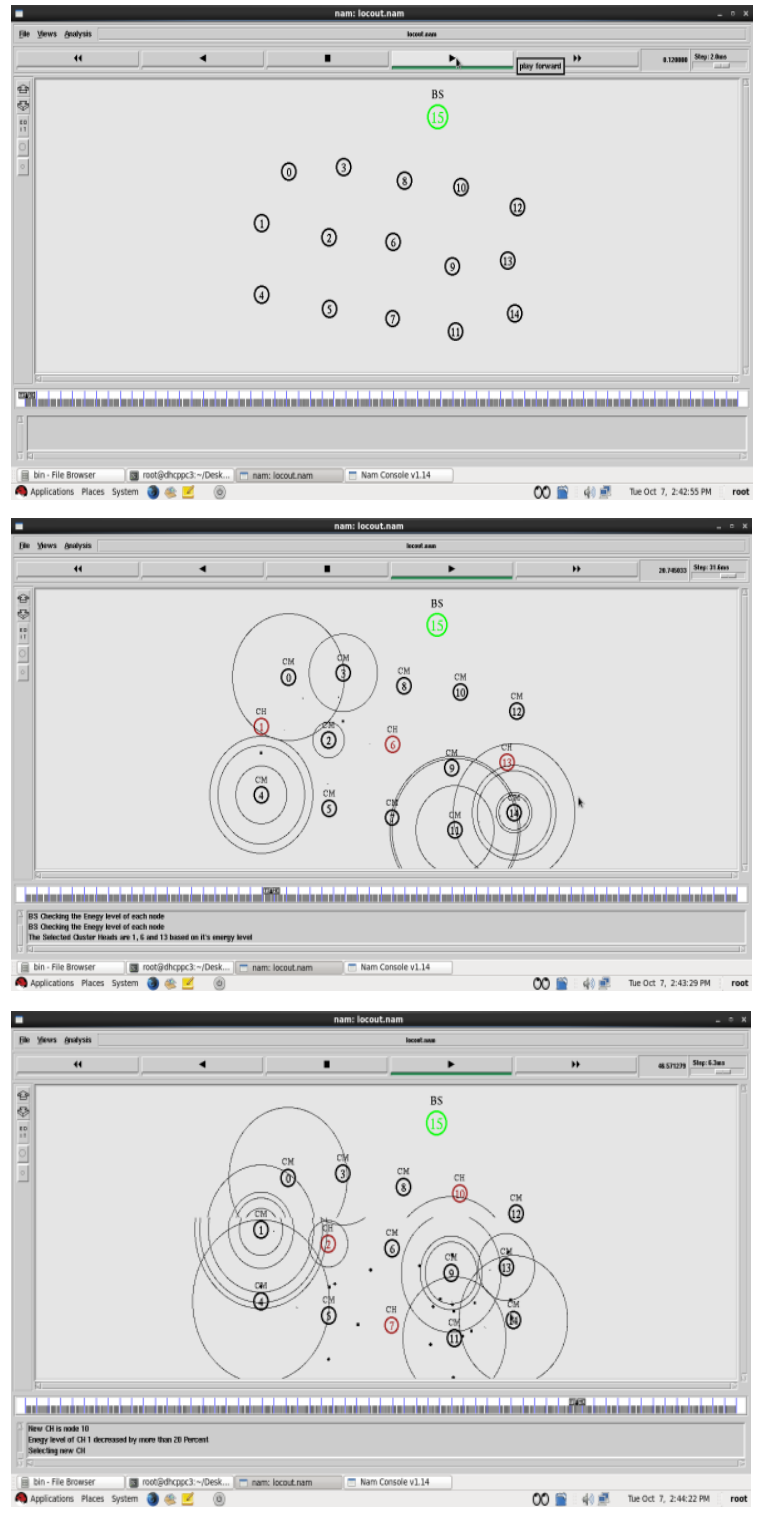

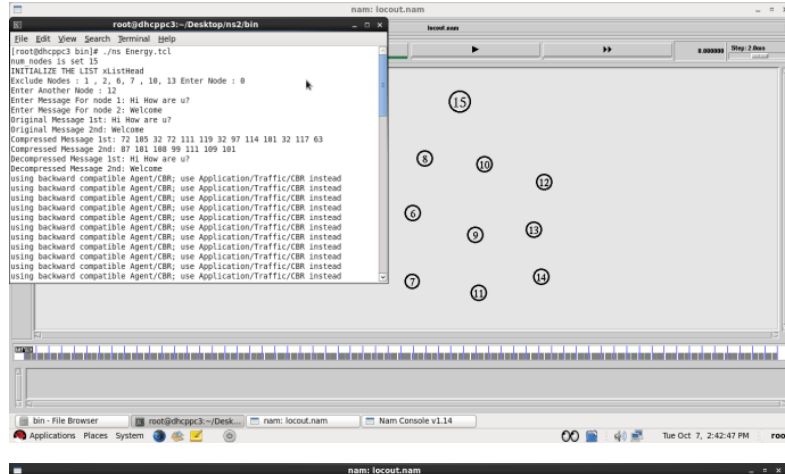
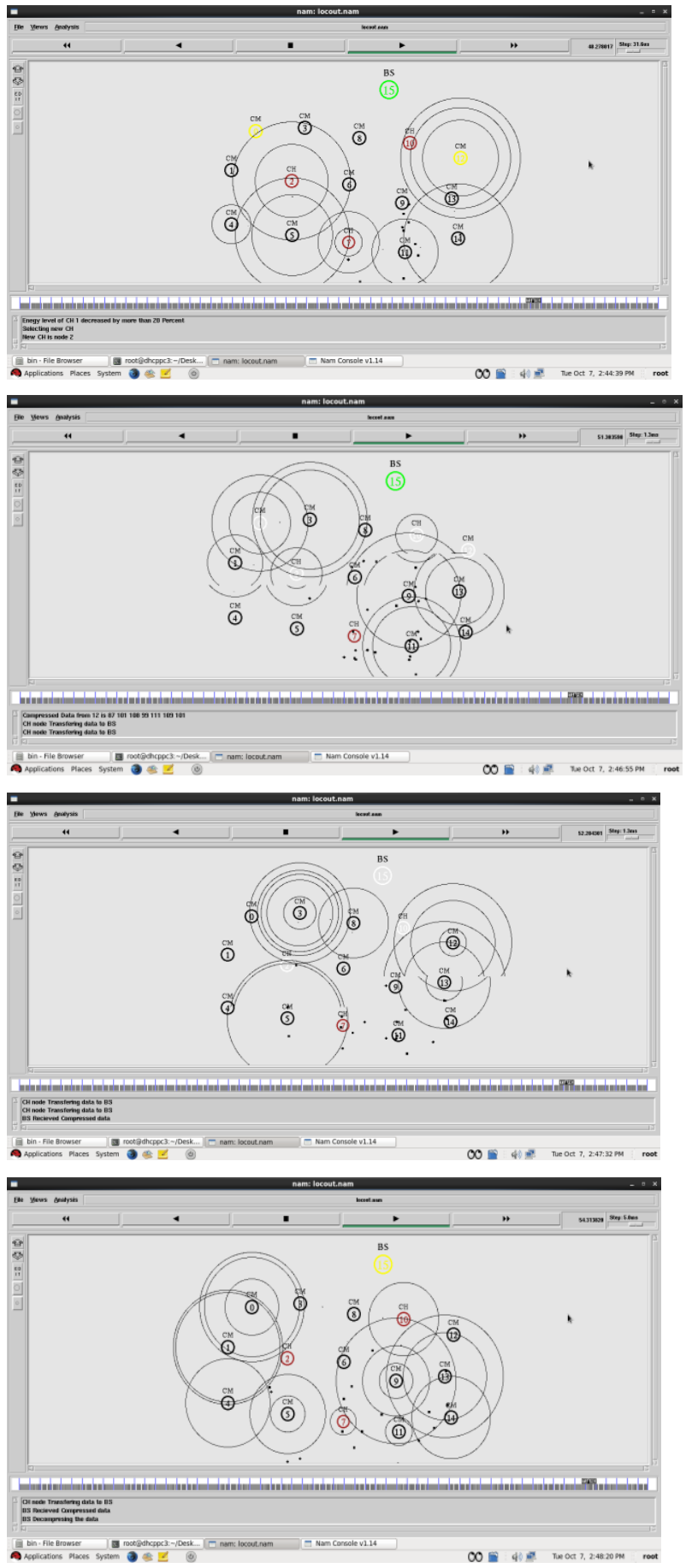

Fig 4: screenshot of node creation, randomized rotation and data compression technique 


\section{PERFORMANCES EVALUATION 3.1 Performances Evaluation of Leach and Leach-c.}

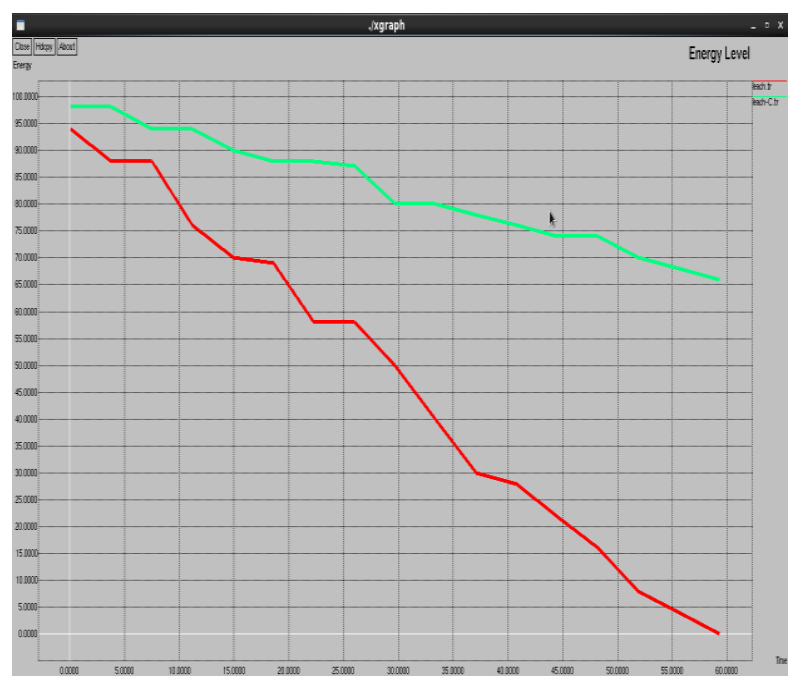

Fig 5: Comparison based on Energy

The leach and leach-c performance are conducted on the parameter of the time and energy. The time is given in the $\mathrm{x}-$ axis and the energy is given in the y-axis. In the Fig 5, the Energy level of the leach and leach-c protocol has been displayed with respect to time. In the initial state both are at the same energy level. In case of leach they perform a poor clustering set up during a given round thus energy level decreases drastically but in case of the leach-c protocol they perform centralized clustering algorithm and also randomized rotation of the cluster head so energy is reduced gradually. Thus leach-c consumes less amount of energy.

\subsection{Performances evaluation between compressed and uncompressed data.}

The time is given in the $\mathrm{x}$-axis and the energy is given in the y-axis.

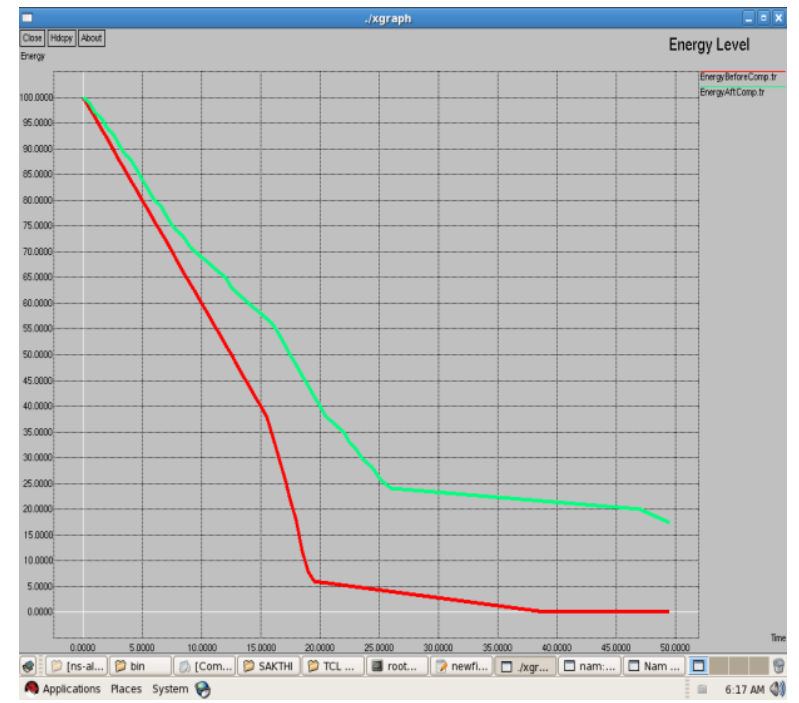

Fig 6: Energy level comparison between compressed and uncompressed data

In the Fig 6, the data is transferred without compression it consumes large amount energy but When the data is transferred with compression they consume only less amount of energy.

\section{CONCLUSION}

The sensor node select their cluster head based on their energy level. The sensor node with higher energy is represented as the cluster head .Implementing leach-c protocol life time of the cluster head has been increased (cluster head performs randomized rotation based on the energy level)and by using data compression technique only less amount of energy has been consumed during the transmission of the data thus the energy is saved and life time of the sensor node has been increased Future scope of the work is to perform data transmission based on the TDMA(Time division multiple access), time slot will be allotted to each sensor node ,hence the respective node can transfer data in a given time rest of the node will be in the sleep mode

\section{REFERENCES}

[1] P. Corke, T. Wark, R. Jurdak, W. Hu, P. Valencia, D. Moore, Environmental wireless sensor networks, Proceedings of the IEEE (2010) 1903-1917.

[2] F. Cheng Jiang , C. Hsing Lin ,H-Wei Wu Lifetime elongation of ad hoc networks under flooding attack using power-saving technique (2013).

[3] W. Heinzelman, A. Sinha, A. Wang, A. Chandrakasan."Energy-Scalable Algorithms and Protocols for WirelessMicrosensor Networks", In Proceedings of the InternationalConference Acoustics, Speech, and Signal Processing (ICASSP '00), June 2003.

[4] M.J. Handy, M. Haase, D. Timmermann, Low energy adaptive clustering hierarchy with deterministic clusterhead selection, in: Proc. Fourth IEEE Conference on Mobile and Wireless Communications Network, 2002, pp. 368-372.

[5] J. Vazifehdan, R. Venkatesha Prasad, and I. Niemegeers, Energy-Efficient Reliable Routing ConsideringResidual Energy in Wireless Ad Hoc Networks (2012)

[6] S. Babaie, A.K. Zadeh, M.G. Amiri, The new clustering algorithm with cluster members bounds for energy dissipation avoidance in wireless sensor network, in: Proc. Computer Design and Applications, ICCDA, June 2010, pp. 613-617.

[7] H.D. Tarigh, M. Sabaei, A new clustering method to prolong the lifetime of WSN, in: Proc. International Conference on Computer Research and Development, ICCRD, March 2011, pp. 143-148.

[8] S. Lindsey, C.S. Raghavendra, PEGASIS: powerefficient gathering in sensor information systems, in: Proc. IEEE Aerospace Conference, Vol. 3, March 2002, pp. 1125-1130.

[9] T. Kanungo, D.M. Mount, N.S. Netanyahu, C.D. Piatko, R. Silverman, A.Y. Wu, An efficient k-means clustering algorithm: analysis and implementation, IEEE Transactions on Pattern Analysis and Machine Intelligence 24 (2002) 881-892.

[10] D Zhang, L Guang, K Zheng, M .Xuechao Ming, , and Zhao-Hua Pan An Energy-Balanced Routing Method Based on Forward-Aware Factor for Wireless Sensor Networks (2013) 